\title{
PAPER \\ Design of Sliding-Mode-Observer-Based Sensorless Control of Boost Converters for High Dynamic Performance
}

\author{
Xutao Li, Minjie Chen and Tsutomu Yoshihara \\ Graduate School of Information, Production and Systems, Waseda University \\ 2-7 Hibikino, Wakamatsu-ku, Kitakyushu, Fukuoka 080-0135, Japan \\ E-mail: leexutao2014@akane.waseda.jp, yoshiharatsutomu@waseda.jp
}

\begin{abstract}
An inductor or switch current is necessary to control a boost converter in the continuous conduction mode for high dynamic performance. Sensorless current control of a boost converter reduces its cost, size, and weight. A Luenberger observer (LO) only feeds a linear output estimation error back to the observer. In this paper, a sliding mode observer (SMO), which feeds a nonlinear output estimation error back to the observer, was proposed for the sensorless control of a boost converter to improve its dynamic performance. Simulations by Simulink and practical experiments on an NJU20010 digital signal processor are performed. It is shown that the proposed SMObased sensorless control of a boost converter maintains better dynamic performance than LO-based sensorless control and conventional sensor-based double-loop Proportional-Integral (PI) control.
\end{abstract}

Keywords: boost converter, digital, sensorless control, sliding mode observer, dynamic performance

\section{Introduction}

For a DC-DC switching converter, a feedback controller is always necessary because variation in the input voltage is usually unavoidable, the load always changes in a range, and the parameters of the components in the circuit vary with the environment. For a boost converter in the continuous conduction mode (CCM), analysis in the frequency domain shows that there is a right-half-plane zero (RHPZ) in the transfer function from the duty ratio to the output voltage [1]. The crossover frequency of the open loop gain is severely restricted by this RHPZ, resulting in poor dynamic performance if single-loop output voltage control is adopted. The time-domain analysis of a sliding-mode-controlled boost converter shows that an unstable inductor current will result if only the output voltage is used [2]. Therefore, either an inductor or a switch current is required for the control of a boost converter in the CCM. This situation is similar to the control of buck-boost and fly-back converters.

Usually, the inductor or switch current is sensed by a shunt resistor with an amplifier, a transformer, or an active filter for the current control of boost converters [3]. However, all the methods unavoidably increase the cost, size, and weight and cause a slight power loss. The sensorless control of boost converters can provide a solution to the above problems. Some sensorless controls have been introduced in previous papers as follows. The use of the integral of the voltage drop on the inductor to estimate the inductor current was introduced in [4], [5], the use of the predicted inductor current for peak current control was introduced in [6], [7], and the use of a state observer based on the state-space equation was introduced in [8], [9]. Investigation results show that, a Luenberger observer (LO) [9] and Kalman filter (KF) [10] are most effective in observing the inductor current of switching converters. However, some drawbacks have also been pointed out as follows. It was shown in [10] that the robustness to parameter uncertainty and external noise cannot be guaranteed for the $\mathrm{LO}$ and $\mathrm{KF}$ and that the realtime implementation of the $\mathrm{KF}$ involves significant numerical complexity. The theoretical analysis in [11] shows that for the LO, the convergence of the estimation error is only ensured in a bounded region. In the formulas of the $\mathrm{LO}$ and $\mathrm{KF}$, a linear output voltage estimation error is fed back to the observer. This means that the estimation error cannot be forced to zero quickly and the convergence accuracy of the observer is not good, as shown in [9], [10].

As presented in [12] - [16], for a sliding mode observer (SMO), a nonlinear output estimation error is fed back to the observer. Provided a bound on the magnitude of the disturbances is known, the SMO can force the estimation error to converge to zero in a finite time, while the observer states converge asymptotically to the actual system states. There have been several studies on the application of the SMO to switching power suppliers [11], [17]-[19]. However, there have been no papers introducing the application of the SMO to the sensorless current control of boost, buck-boost, and fly-back converters with a clear description of the SMO. Therefore, in this paper, an improved discrete SMO is proposed for the sensorless control of boost converters to improve their dynamic 
performance. Simulations by Simulink and practical experiments on an NJU20010 digital processor are performed. It is shown that the dynamic performance of the boost converter is greatly improved by the proposed SMO-based control compared with LO-based control and conventional sensor-used double-loop PI control.

\section{Problem Formulation}

\subsection{Problems of the conventional sensor-based current control of boost converters}

As shown in Fig. 1, for a single-loop output-voltagecontrolled boost converter in the CCM, neither a linear controller such as a PID [20] nor a nonlinear controller such as a sliding mode controller [21] can maintain good dynamic performance because of the RHPZ in the transfer function from the duty ratio to the output voltage. Doubleloop current control [20] or a controller using the inductor current as a system state in the time domain [21] is widely adopted to maintain good dynamic performance; however, the inductor or switch current should be sensed, as shown in Fig. 2, which illustrates a double-loop current control. Although there are many methods of detecting the inductor or switch current [3], all the methods unavoidably increase the cost, size, and weight and cause a slight power loss. This is similar to the control of buck-boost and fly-back converters.

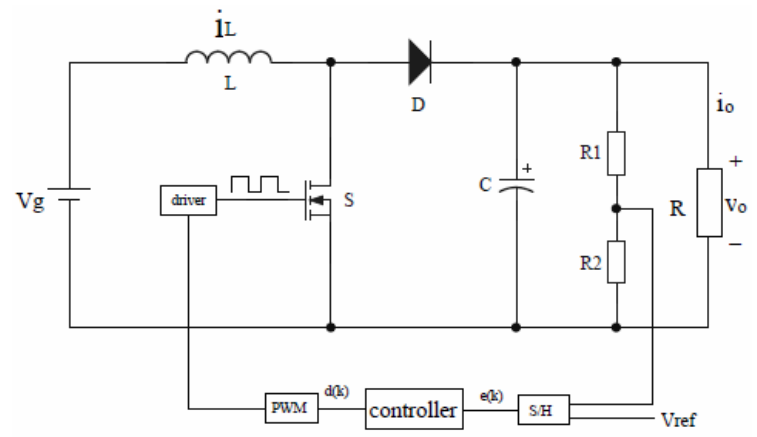

Fig. 1 Single-loop output-voltage-controlled boost converter

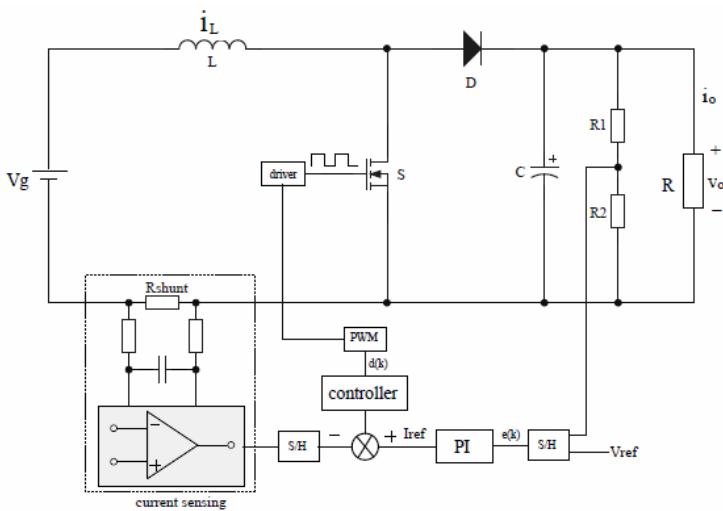

Fig. 2 Conventional double-loop-controlled boost converter

\subsection{Problem formulation of the sensorless control of boost converters}

The sensorless control of boost converters can provide a solution to the above problems caused by current sensing. The sensorless control of boost converters is shown in Fig. 3 , in which some parasitic parameters are considered. The inductor current is first estimated using the duty ratio, the input voltage, and the output voltage, then the estimated inductor current is applied to the controller. From the inductor current estimation methods introduced in [4] - [9], the following LO is the most effective method of observing the inductor current of switching mode power suppliers.

$$
\begin{aligned}
& \dot{\hat{x}}=\Phi(\hat{x})+\Gamma(\hat{x}, u)+K(y-\hat{y}) \\
& \hat{y}=C \hat{x}(t)
\end{aligned}
$$

here $\hat{x}$ is the estimate of the system state $x$, and $y$ is the measurable output. The LO can be a nonlinear [9] or linear observer [8] depending on the constructed model of the control object. However, it can be seen that the error between the control system output $y$ and the observer output $\hat{y}$ is fed linearly back to the observer. As a result, the estimation error cannot be forced to zero quickly, and the convergence accuracy of the observer deteriorates in the presence of unknown disturbances [9], [10].

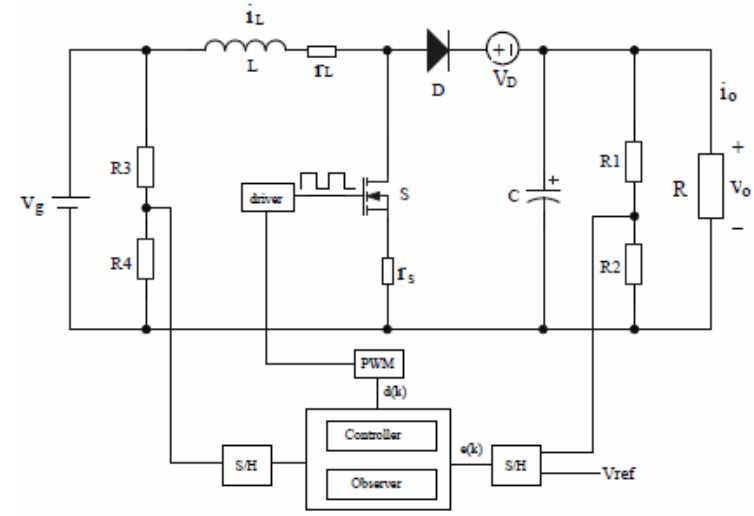

Fig. 3 Sensorless controlled boost converter

As expressed by Eq. (2), through feeding the output estimation error nonlinearly back to the observer, the SMO provides a method of improving the observation of the system state. Provided a bound on the magnitude of the disturbances is known, the SMO can force the output estimation error to converge to zero in a finite time, while the observer states asymptotically converge to the actural system states.

$$
\begin{aligned}
& \dot{\hat{x}}=\Phi(\hat{x})+\Gamma(\hat{x}, u)+L \operatorname{sgn}(y-\hat{y}) \\
& \hat{y}=C \hat{x}(t)
\end{aligned}
$$

Although there have been several studies on the application of the SMO to switching mode power suppliers [11], [17] - [19], there have been no papers introducing the application of the SMO to the sensorless control of boost converters with a clear description of the SMO. Therefore, in this paper, an improved SMO in discrete time is proposed for the sensorless control of boost converters to improve their dynamic performance. 


\section{Introduction of the Proposed Sliding Mode Observer}

Suppose that a discrete linear time-invariant system has the form

$$
\begin{aligned}
& x(k+1)=\Phi x(k)+\Gamma u(k)+F \xi(k) \\
& y(k)=C x(k)
\end{aligned}
$$

where $x(k) \in R^{n}$ is the state, $y(k) \in R^{p}$ is the measurable output, $u(k) \in R^{m}$ is the known input, $\xi(k) \in R^{q}$ is the bounded disturbance, $q \leq p$, and $\Phi, \Gamma, F$, and $C$ are constant matrices of appropriate dimensions. $\zeta(k)$ is unknown but assumed to be bounded and satisfy

$$
\|\xi(k)\| \leq \rho
$$

where $\rho$ is known. To construct state $x(k)$ in the system given by Eq. (3), an improved SMO is proposed.

\subsection{Proposed sliding mode observer}

The proposed SMO for the system in Eq. (3) is written as

$$
\begin{aligned}
& \hat{x}(k+1)=\Phi \hat{x}(k)+\Gamma u(k)+G_{l} e_{y}(k)+G_{n} v \\
& \hat{y}(k)=C \hat{x}(k)
\end{aligned}
$$

where

$$
e_{y}(k)=y(k)-\hat{y}(k), v=\operatorname{sgn}\left(e_{y}\right)
$$

$\hat{x}(k)$ is the estimate of state $x(k), v$ is a discontinuous feedback compensation, which can eliminate the effect of the nonlinear term $\zeta(k)$ in Eq. (3), and, when combined with $u(k)$, it guarantees the asymptotic stability of error estimation. The constant matrix $G_{n}$ is the observer gain matrix, which guarantees that a sliding mode is attained on the sliding surface in the error space of the state estimation. Compared with Eq. (2), a linear output error term $G_{l} e_{y}$ is added to the observer in order to enlarge the sliding mode region.

\subsection{Solvability conditions of the proposed SMO}

The necessary and sufficient conditions of the SMO given by Eq. (5) for the system in Eq. (3) are as follows:

I. $\operatorname{rank}(C F)=\operatorname{rank}(F)$.

II. The invariant zeros of the triple $(\Phi, F, C)$ lie inside the unit circle.

\subsection{Observation error dynamics}

We define $e(k)=x(k)-\hat{x}(k)$ as the state estimation error. From Eqs. (3) and (5), the reconstruction error is given as follows:

$$
\begin{aligned}
& e(k+1)=\left(\Phi-G_{l} C\right) e(k)+F \xi(k)-G_{n} v \\
& e_{y}(k)=C e(k)
\end{aligned}
$$

We define the sliding surface as

$$
s=\left\{e(k) \mid e_{y}(k)=0\right\}
$$

As proved in [12], [16], the error system given by Eqs. (6) and (7) is asymptotically stable, and a convergent sliding-mode motion occurs on the sliding surface defined in Eq. (8) under some conditions. Assuming that the ideal sliding mode of the system in Eq. (6) is obtained after a finite time $k$, at which $e_{y}(k)=0$, the equivalent control at the sliding stage is obtained as

$$
\nu_{e q}(k)=\left(C G_{n}\right)^{-1}(C \Phi e(k)+C F \xi(k))
$$

Substituting Eq. (9) into the state error equation Eq. (6) gives the reduced-order system

$$
e(k+1)=\left(I-G_{n}\left(C G_{n}\right)^{-1} C\right)(\Phi e(k)+F \xi(k))
$$

Ideally, the error system in Eq. (6) will converge to the origin along the sliding surface in Eq. (8) under the control expressed as Eq. (9).

\subsection{Parameter determination of the SMO in Eq. (5)}

To determine the parameters $G_{l}$ and $G_{n}$ in Eq. (5), the application of a linear transformation to change the coordinates of the system in Eq. (3) was introduced in [13], [16], and the use of a linear matrix inequality was introduced in [22]. In this paper, a simple method introduced in [12] is proposed as follows.

As one of the eigenvalues of $\left(I-G_{n}\left(C G_{n}\right)^{-1} C\right) \Phi$ is zero, $G_{n}$ can be determined through assigning the other $n-1$ eigenvalues. Moreover, if there exists a real number such that

$$
F=\eta G_{n}
$$

and all the eigenvalues of $\left(I-G_{n}\left(C G_{n}\right)^{-1} C\right) \Phi$ lie inside the unit circle on the discrete complex plane, it can be found that $e(k)$ is independent of $\zeta(k)$ through substituting Eq. (11) into Eq. (10). That is, the sliding motion given by Eq. (10) is stable and will not be affected by any disturbance.

We define a discrete Riccati equation as follows:

$$
\Phi P \Phi^{T}-\frac{1}{\alpha+C P C^{T}} \Phi P C^{T} C P \Phi^{T}-P=-Q
$$

where $Q$ is a positive semi-definite symmetric matrix and $\alpha$ is a positive real number. Solving Eq. (12) to find the unique positive definite symmetric matrix $P$, the matrix $G_{l}$ is given by

$$
G_{l}=\frac{1}{\alpha+C P C^{T}} \Phi P C^{T}
$$




\section{Design of the Proposed SMO-Based Sensorless Control for Boost Converters}

\subsection{Construction of discrete AC small-signal average value model of boost converters}

The boost converter used in this paper is shown in Fig. 3, in which several parasitic components are considered. Referring to [1], the AC average value model of the boost converter in the CCM is constructed as follows:

$$
\dot{x}=A_{c} x+B_{c} d+E_{c} w
$$

where

$$
\begin{aligned}
A_{c} & =\left[\begin{array}{cr}
-\frac{r_{L}+D r_{s}}{L} & -\frac{D^{\prime}}{L} \\
\frac{D^{\prime}}{C} & 0
\end{array}\right] \\
B_{c} & =\left[\begin{array}{cc}
\frac{V_{g}}{D^{\prime} L}-\frac{\left(r_{L}+r_{s}\right) V_{r e f}}{D^{\prime 2} R L} \\
-\frac{V_{r e f}}{D^{\prime} R C}
\end{array}\right] \\
E_{c} & =\left[\begin{array}{cc}
\frac{1}{L} & 0 \\
0 & -\frac{1}{C}
\end{array}\right] \\
D^{\prime} & =\frac{\left(r_{s} V_{r e f}+R V_{g}\right)\left(1+\sqrt{\left.1-\frac{4 R\left(r_{L}+r_{s}\right)\left(V_{r e f}+V_{D}\right) V_{r e f}}{\left(r_{s} V_{r e f}+R V_{g}\right)^{2}}\right)}\right.}{2 R\left(V_{r e f}+V_{D}\right)}
\end{aligned}
$$

$D$ is the duty ratio in one switching period and $D^{\prime}=1-D . x$ $=\left[\begin{array}{ll}i_{L} & v_{o}\end{array}\right]^{T}$ and $w=\left[\begin{array}{ll}v_{g} & i_{o}\end{array}\right]^{T}$ are the corresponding AC small signals. Here, although $v_{o}$ and $v_{g}$ are not scaled by the corresponding dividing resistors shown in Fig. 3, the parameters of the controllers will be scaled at the execution stage on a digital processor.

To design a discrete SMO and controller, the above state-space Eq. (14) should be discretized, and the discrete counterpart is written as

$$
x(k+1)=A_{d} x(k)+B_{d} d(k)+E_{d} w(k)
$$

where

$$
A_{d}=e^{A_{c} T}, B_{d}=\int_{0}^{T} e^{A_{c} \tau} d \tau B_{c}, E_{d}=\int_{0}^{T} e^{A_{c} \tau} d \tau E_{c}
$$

On the basis of Eq. (15), the SMO is designed as described in the following section.

\subsection{Construction of the sliding mode observer}

To design the SMO, we define

$$
x(k)=\left[\begin{array}{c}
i_{L}(k) \\
v_{o}(k)
\end{array}\right], u(k)=\left[\begin{array}{c}
d(k) \\
v_{g}(k)
\end{array}\right], \xi(k)=\left[i_{o}(k)\right]
$$

First, we change Eq. (15) into the following form:

$$
\left\{\begin{array}{l}
x(k+1)=\Phi x(k)+\Gamma u(k)+F \xi(k) \\
y(k)=\left[\begin{array}{ll}
0 & 1
\end{array}\right] x(k)
\end{array}\right.
$$

where

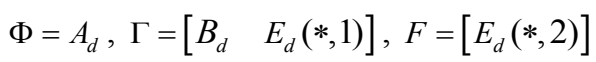

Then, the SMO is constructed as follows:

$$
\left[\begin{array}{c}
\hat{i}_{L}(k+1) \\
\hat{v}_{o}(k+1)
\end{array}\right]=\Phi\left[\begin{array}{c}
\hat{i}_{L}(k) \\
\hat{v}_{o}(k)
\end{array}\right]+\Gamma u(k)+G_{l} e_{y}(k)+G_{n} v
$$

where

$$
e_{y}(k)=y(k)-\hat{y}(k), v=\operatorname{sgn}\left(e_{y}\right)
$$

\subsection{Design of SMO-based sliding mode controller}

The SMO in Eq. (17) can be used for the average-valuemodel based sensorless control of a boost converter, such as double-loop PI control and state feedback control. As double-loop PI control is well known to almost all power electronics engineers and researchers, in this paper, the design of a sliding mode controller, which can maintain better dynamic performance than a linear controller, is introduced in the following.

First, we change Eq. (15) into the following form:

$$
x(k+1)=A_{d} x(k)+B_{d} d(k)+E_{1} v_{g}(k)+E_{2} i_{o}(k)
$$

where

$$
E_{1}=E_{d}(*, 1), E_{2}=E_{d}(*, 2)
$$

Then, the system state variables are selected as follows to design a discrete sliding mode controller. Both the inductor current error and the output voltage error are used to improve the accuracy of the output voltage.

$$
x(k)=\left[\begin{array}{c}
x_{1}(k) \\
x_{2}(k) \\
x_{3}(k)
\end{array}\right]=\left[\begin{array}{c}
i_{L}(k)-i_{r e f}(k) \\
v_{o}(k) \\
\sum_{1}^{k} x_{1}+\sum_{1}^{k} x_{2}
\end{array}\right]
$$

here $i_{R e f}$ represents the instantaneous reference inductor current and is generated from the following PI controller:

$$
i_{r e f}(k)=k_{P} v_{o}(k)+k_{I} \sum_{j=1}^{k} v_{o}(j)
$$


We define the sliding surface as

$$
s(k)=c_{1} x_{1}(k)+c_{2} x_{2}(k)+c_{3} x_{3}(k)
$$

We suppose that $i_{o} \leq \rho$ at all times, where $\rho$ represents the maximum disturbance from the load. Using the equivalent control $s(k+1)=s(k)$ at the sliding stage, the equivalent control can be obtained from Eqs. (18) - (20) and is written as follows through replacing $x(k)$ by $\hat{x}(k)$ in Eq. (17):

$$
d_{e q}=-\left(M B_{d}\right)^{-1}\left[\left(M A_{d}-N\right) \hat{x}(k)+M E_{1} v_{g}+M E_{2} \rho-c_{3} i_{R e f}(k)\right](21)
$$

where

$$
\begin{array}{ll}
M & =\left[\begin{array}{ll}
c_{1}+c_{3} & -\left(k_{P}+k_{I}\right)\left(c_{1}+c_{3}\right)+c_{2}+c_{3}
\end{array}\right] \\
N & =\left[\begin{array}{ll}
c_{1} & -k_{P}\left(c_{1}+c_{3}\right)+c_{2}
\end{array}\right]
\end{array}
$$

The actual trajectory of a sliding-mode-controlled system is shown in Fig. 4. The equivalent control term in Eq. (21) forces the system states to move toward the origin at the sliding stage. A switching control term should also be used to bring the system states onto the sliding surface $s$ and trap them into a small range of $s$ at the sliding stage. Although a sign function can ideally realize sliding mode control, the chattering problem will also appear [24]. In fact, only quasi-sliding mode control is feasible in practical applications. Therefore, the switching control term in Eq. (22), which is a saturation function, is adopted to eliminate the chattering at the steady stage.

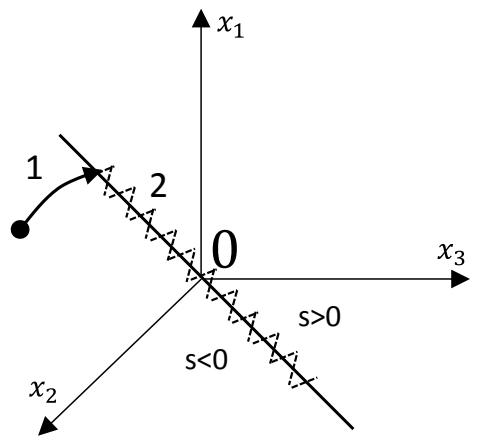

Fig. 4 Trajectory of a sliding-mode-controlled system

$$
d_{s w}(k)=-\beta \operatorname{sat}\left(\frac{s}{\Delta}\right)=\left\{\begin{array}{cc}
-\beta & s>\Delta \\
-\frac{\beta}{\Delta} s & |s| \leq \Delta \\
\beta & s<\Delta
\end{array}\right.
$$

here $\Delta$ represents the boundary layer around the sliding surface $s$. The overall discrete sliding mode controller is written as

$$
d(k)=d_{e q}+d_{s w}
$$

The derivation of the stability conditions of the discrete sliding mode controller in Eq. (23) is given in [21], [23]. The parameter determination of the sliding mode controller is also presented in [23], and an empirical approach to selecting the sliding coefficients is presented in [21].

\section{Simulations and Experiments}

Table 1 Parameters of a boost converter

\begin{tabular}{l|l}
\hline Input voltage: $v_{g}=10 \mathrm{~V}$ & Output voltage: $v_{o}=20 \mathrm{~V}$ \\
\hline Capacitor: $C=1000 \mathrm{uF}$ & Inductor: $\mathrm{L}=47 \mathrm{uH}, \mathrm{DCR}=24 \mathrm{~m} \Omega$ \\
\hline Load: $\mathrm{R}=25 \Omega$ & Switching frequency: $f_{s}=150 \mathrm{kHz}$ \\
\hline Switch: $r_{O N}=36 \mathrm{~m} \Omega$ & Diode: $V_{\text {Drop }}=1.25 \mathrm{~V}$ \\
\hline
\end{tabular}

To verify the improved dynamic performance by the proposed SMO-based sensorless control, a boost converter whose parameters are listed in Table 1 is used.

\subsection{Simulations of SMO-based and LO-based sensorless sliding mode control of the boost converter}

Substituting the parameters in TABLE 1 into Eq. (14), the discrete counterpart in Eq. (15) is obtained as follows:

$$
\begin{aligned}
A_{d} & =\left[\begin{array}{rr}
0.9938 & -0.0661 \\
0.0031 & 0.9999
\end{array}\right], B_{d}=\left[\begin{array}{r}
2.9965 \\
-0.0067
\end{array}\right] \\
E_{d} & =\left[\begin{array}{lr}
0.1414 & 0.0002 \\
0.0002 & -0.0067
\end{array}\right]
\end{aligned}
$$

Referring to [21], [23], the sliding mode controller in Eq. (23) is obtained as

$$
\begin{array}{r}
d(k)=-0.0759 \hat{i}_{L}(k)-0.0362 \hat{v}_{o}(k)-0.0507 v_{g}+ \\
0.0580 i_{r e f}(k)+0.0343-0.12 \operatorname{sat}\left(\frac{s(k)}{0.1}\right)
\end{array}
$$

where

$$
\begin{aligned}
s(k) & =0.5 x_{1}(k)+5 x_{2}(k)+0.1 x_{3}(k) \\
i_{\text {ref }}(k) & =-10.016 v_{o}(k)-0.0053 \sum_{j=1}^{k} v_{o}(j)
\end{aligned}
$$

$\hat{\imath}_{L}$ and $\hat{v}_{o}$ are the estimated states obtained by the SMO in Eq. (17).

Firstly, we determine the parameter $G_{l}$ in Eq. (17). Setting $Q=I_{2}$ and $\alpha=1$, where $I_{2}$ is a unit matrix, the matrix $P$ in Eq. (12) is obtained as

$$
P=\left[\begin{array}{rr}
76.6166 & 0.3167 \\
0.3165 & 1.6196
\end{array}\right]
$$


Substituting the above $P$ into Eq. (13), the matrix $G_{l}$ in Eq. (17) is obtained as $G_{l}=\left[\begin{array}{ll}0.0793 & 0.6186\end{array}\right]^{\mathrm{T}}$, and the eigenvalues of $\Phi-G_{l} C$ are 0.9938 and 0.0448 .

Secondly, we determine the parameter $G_{n}$ in Eq. (17). Substituting Eq. (11) into Eq. (10), it is found that the eigenvalues of $\left(I-G_{n}\left(C G_{n}\right)^{-1} C\right) \Phi$ are 0.9939 and 0 . Therefore, there exists a real number $\eta$ such that the sliding motion given by Eq. (10) is stable and will not be affected by any disturbance. Selecting $\eta=0.8$, the matrix $G_{n}$ is obtained as $G_{n}=\left[\begin{array}{ll}0.0003 & -0.0083\end{array}\right]^{\mathrm{T}}$. Then, the SMO in Eq. (17) is given by

$$
\begin{aligned}
x(k+1) & =\left[\begin{array}{rr}
0.9938 & -0.0661 \\
0.0031 & 0.9999
\end{array}\right] x(k)+\left[\begin{array}{rr}
2.9965 & 0.1414 \\
-0.0067 & 0.0002
\end{array}\right] u(k) \\
& +\left[\begin{array}{r}
0.0793 \\
0.6186
\end{array}\right] e_{y}(k)+\left[\begin{array}{r}
0.0003 \\
-0.0083
\end{array}\right] v(k)
\end{aligned}
$$

where $x(k+1)=\left[\begin{array}{ll}\hat{l}_{L}(k+1) & \hat{v}_{o}(k+1)\end{array}\right]^{T}$.

Similarly, $\alpha$ in Eq. (12) and $\eta$ in Eq. (11) can be set to other values, e.g., $\alpha=0.1, \alpha=5$ and $\eta=0.2$, to investigate the convergence performance of the SMO. Simulations show that good convergence performance is maintained by $\alpha=1$ and $\eta=0.8$. The dynamic response of the boost converter whose parameters are listed in Table 1 controlled by the sliding mode controller in Eq. (24) and the SMO in Eq. (25) is shown in Fig. 5.

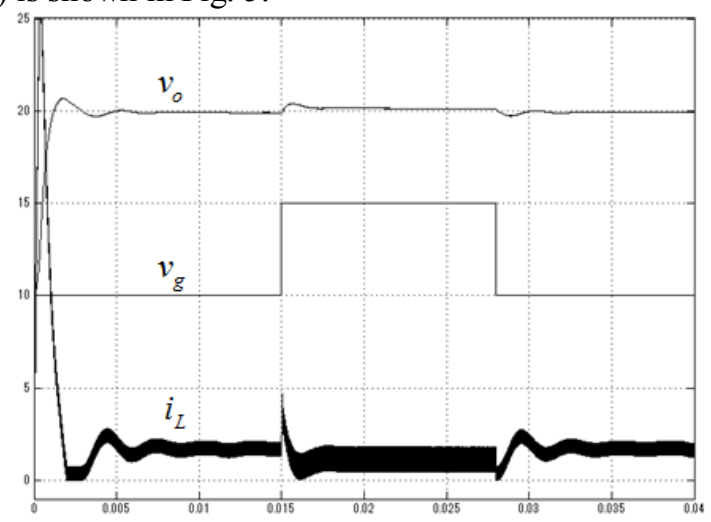

(a) Dynamic response to an input voltage disturbance

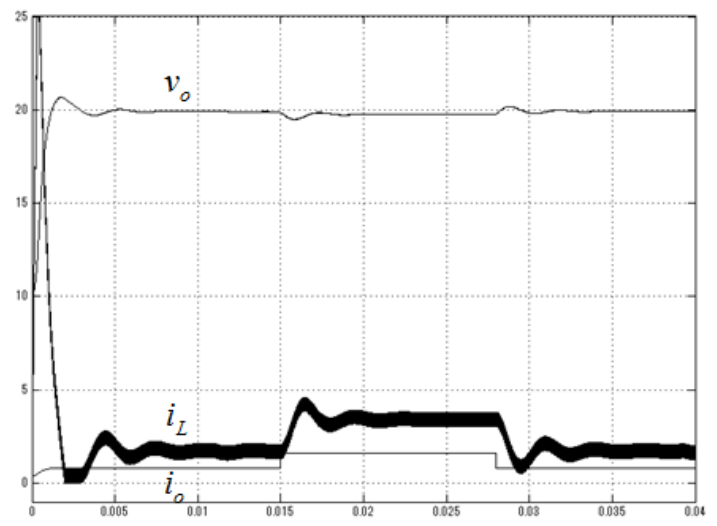

(b) Dynamic response to a load disturbance

Fig. 5 Dynamic response of SMO-based sliding mode control

For the LO in Eq. (1), the estimation error dynamics are governed by the eigenvalues of the matrix $\Phi-K C$. The eigenvalue assignment of $\Phi-K C$ is usually used to determine the parameter $K$, and eigenvalues with a large real part and small imaginary part will result in better performance, while those with a small real part and large imaginary part will result in poorer performance. Simulations show that good convergence performance is maintained by the eigenvalues of $\Phi-K C$ of $\{0.8 \pm \mathrm{j} 0.2\}$ and the LO is given in Eq. (26). The dynamic response of the boost converter with the parameters listed in Table 1 controlled by the sliding mode controller in Eq. (24) and the LO in Eq. (26) is shown in Fig. 6.

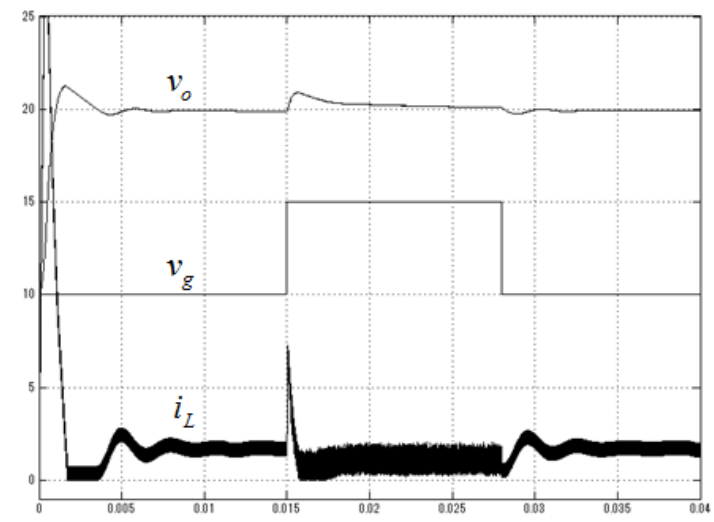

(a) Dynamic response to an input voltage disturbance

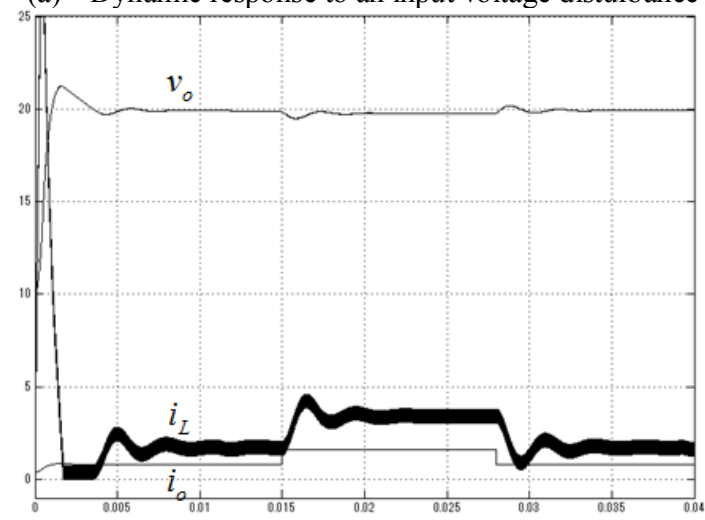

(b) Dynamic response to a load disturbance

Fig. 6 Dynamic response of LO-based sliding mode control

$$
\begin{aligned}
x(k+1) & =\left[\begin{array}{rr}
0.9938 & -0.0661 \\
0.0031 & 0.9999
\end{array}\right] x(k)+\left[\begin{array}{rr}
2.9965 & 0.1414 \\
-0.0067 & 0.0002
\end{array}\right] u(k) \\
& +\left[\begin{array}{r}
215.7881 \\
1.5937
\end{array}\right] e_{y}(k)
\end{aligned}
$$

where $x(k+1)=\left[\begin{array}{ll}\hat{\imath}_{L}(k+1) & \hat{v}_{o}(k+1)\end{array}\right]^{T}$.

From the above simulations, it can be observed that the dynamic response of the boost converter whose parameters are listed in Table 1 is clearly improved by SMO-based sensorless sliding mode control compared with LO-based control when the input voltage is disturbed. Figures. 5(b) and 6(b) show that the dynamic response is very short when the load is disturbed, so that the improvement in the dynamic response is relatively slight when the load is disturbed.

Journal of Signal Processing, Vol. 19, No. 6, November 2015 


\subsection{Practical experiments of LO- and SMO-based sliding mode control of the boost converter}

In the practical experiments, an NJU20010 16-bit digital signal processor produced by NJR Corporation is used to execute the controllers. The limit of the duty ratio is set to $0.05-0.88$. The slew rates of the load current and the input voltage are $250 \mathrm{~mA} / \mu \mathrm{s}$ and $2.0 \mathrm{~V} / \mu \mathrm{s}$, respectively.

The dynamic responses of the boost converter controlled by the sliding mode controller in Eq. (24) with the SMO in Eq. (25) and the LO in Eq. (26) are shown in Figs. 7 and 8, respectively. It can be seen that the overshoot/undershoot and the recovery time are clearly improved when the input voltage is disturbed by the SMO-based control. Also, since the dynamic response to the load disturbance is very short, the dynamic response improvement is relatively slight.

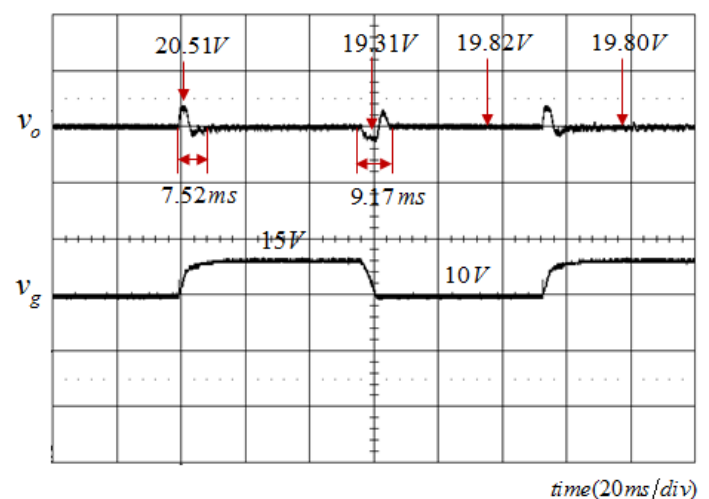

(a) Dynamic response to an input voltage disturbance

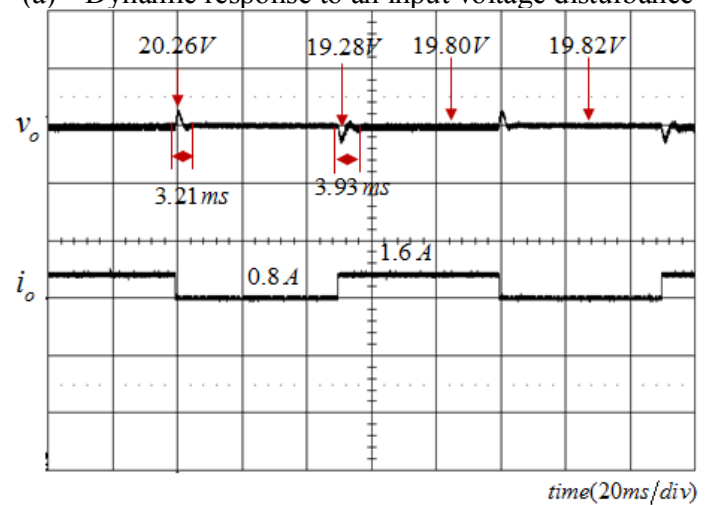

(b) Dynamic response to a load disturbance

Fig. 7 Experimental results of SMO-based sliding mode control

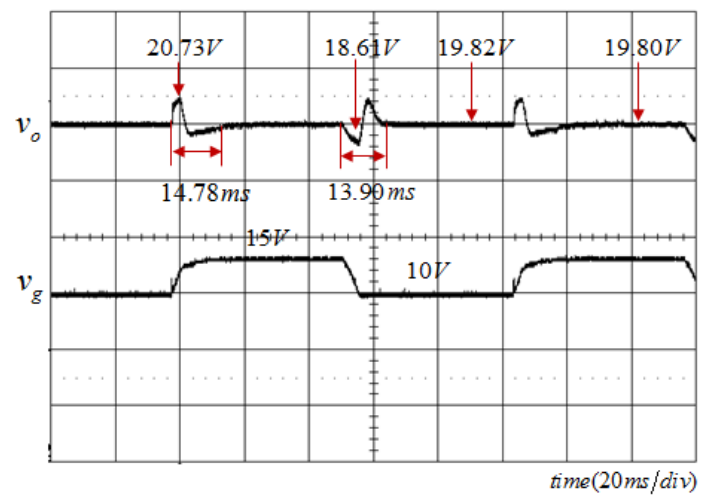

(a) Dynamic response to an input voltage disturbance

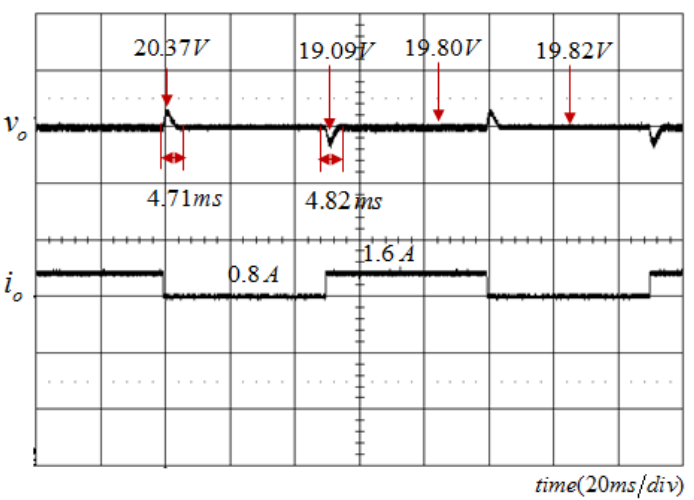

(b) Dynamic response to a load disturbance

Fig. 8 Experimental results of LO-based sliding mode control

\subsection{Comparison with double-loop PI control}

To compare the dynamic performance of the boost converter whose parameters are listed in Table 1 by SMObased sliding mode control and conventional sensor-based double-loop PI control, a double-loop control given in Eqs. (27) and (28), is adopted, in which the inductor current is sensed by a shunt resistor. The counterpart of Eqs. (27) and (28) in continuous time shows that the inner open loop has a gain crossover frequency of $40.8 \mathrm{kHz}$ with a phase margin of $86.2^{\circ}$, and the outer open loop has a gain crossover frequency of $2.47 \mathrm{kHz}$ with a phase margin of $79.8^{0}$ and a gain margin of $18.0 \mathrm{~dB}$. It can be said that the double-loop controller is very good. Figure. 9 shows its dynamic response.

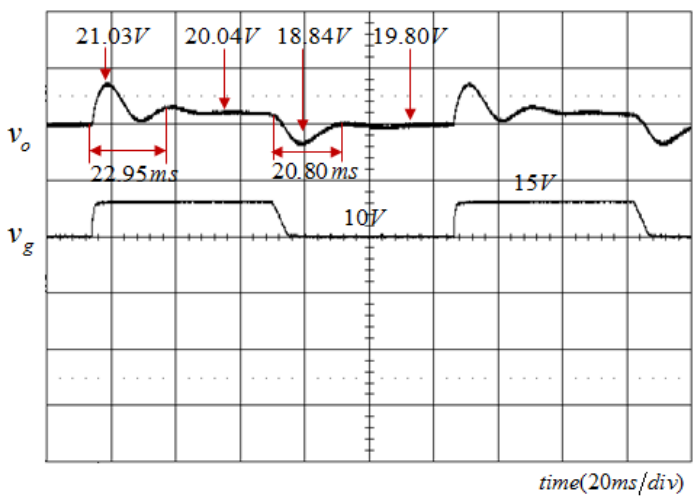

(a) Dynamic response to an input voltage disturbance

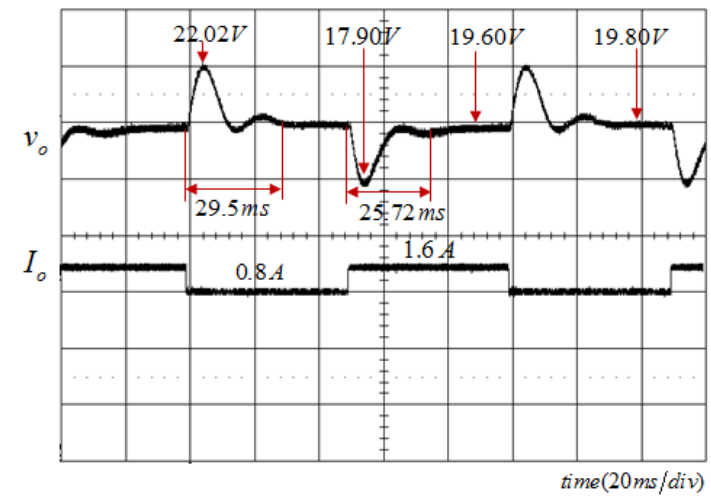

(b) Dynamic response to a load disturbance

Fig. 9 Experimental results of sensor-based double-loop PI control 


$$
\begin{gathered}
d(k)=-2.156 e_{I}(k)-0.00067 \sum e_{I}(j) \\
i_{r e f}(k)=-10.016 v_{o}(k)-0.0053 \sum v_{o}(j)
\end{gathered}
$$

where $e_{I}(k)=i_{L}(k)-i_{r e f}(k)$ and $i_{L}$ is the inductor current, and $i_{\text {ref }}$ is the reference current.

Fig. 10 shows the actual experimental environment.

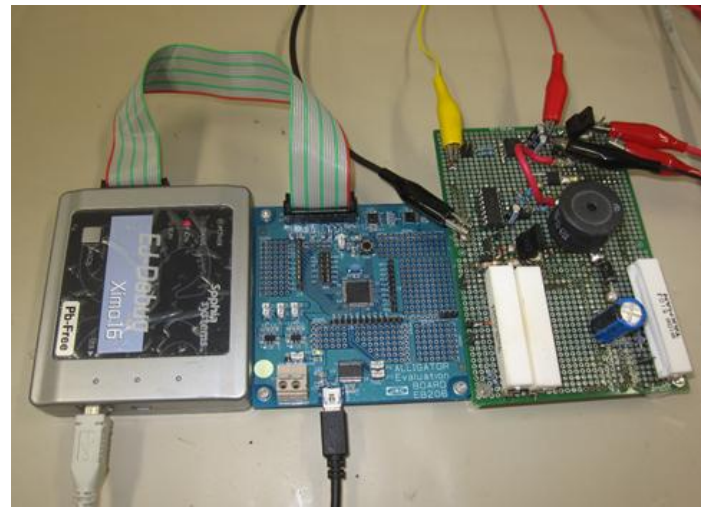

Fig. 10 Actual experimental environment

\subsection{Summary}

The dynamic responses of the boost converter with parameters listed in Table 1 controlled by SMO- and LObased sliding mode controllers are shown in Figs. 5 - 8 . The dynamic response is improved by SMO-based sliding mode control compared with LO-based control. The dynamic response by conventional sensor-based doubleloop PI control is shown in Fig. 9. It shows that the dynamic response is greatly improved by the SMO-based sliding mode control compared with double-loop sensorbased PI control.

\section{Conclusions}

An inductor or switch current is required for the current control of a boost converter in the CCM. A state observer can be used to estimate the inductor current instead of a current-sampling network. An LO linearly feeds the output estimation error back to the observer. An SMO feeds the output estimation error back to the observer via a nonlinear term so that better convergence performance can be maintained. Applying the proposed discrete SMO based sensorless control of a boost converter, simulations and experiments show that the dynamic response was greatly improved compared with LO-based sensorless control and conventional sensor-based double-loop PI control.

\section{References}

[1] R. W. Erickson and D. Maksimovic: Fundamentals of Power Electronics, Kluwer Academic Publishers, 2001.

[2] H. Sira-Ramirez: Sliding motions in bilinear switched networks, IEEE Trans. Circ. Syst., Vol. CAS-34, No. 8, pp. 919-933, 1987.

[3] H. P. Forghani-Zadeh and G. A. Rincón-Mora: Currentsensing techniques for DC-DC Converters, Midwest Symposium on Circuits and Systems, Vol. 2, pp. 577-580, 2002.
[4] P. Midya, P. T. Krein and M. F. Greuel: Sensorless current mode control - An observer-based technique for DC-DC converters, IEEE Trans. Power Elect., Vol. 16, No. 4, pp. 522-526, 2001.

[5] J. T. Mossoba and P. T. Krein: Design and control of sensorless current mode DC-DC converters, Applied Power Electronics Conference and Exposition, Vol. 1, pp. 315$321,2003$.

[6] A. Kelly and K. Rinne: Sensorless current-mode control of a digital dead-beat DC-DC converter, Applied Power Electronics Conference and Exposition, Vol. 3, pp. 17901795, 2004.

[7] Q. Tong, Q. Zhang, R. Min, X. Zou, Z. Liu and Z. Chen: Sensorless predictive peak current control for boost converter using comprehensive compensation strategy, IEEE Trans. Indus. Elect., Vol. 61, No. 6, pp. 1-11, 2013.

[8] A.G.Beccuti, S. Mariethoz, S. Cliquennois., S. Wang and M. Morari: Explicit model predictive control of DC-DC switched-mode power supplies with extended Kalman filtering, IEEE Trans. Indus. Elect., Vol. 56, No. 6, pp 1864-1874, 2009.

[9] G. Cimini., G. Ippoliti, G. Orlando and M. Pirro: Current sensorless solution for PFC boost converter operating both in DCM and CCM, Control \& Automation (MED), pp. 137-142, 2013.

[10] Y. C. Zhang, Z. M. Zhao, T. Lu, L. Q. Yuan, W. Xu and J. G. Zhu: A comparative study of Luenberger observer, sliding mode observer and extended Kalman filter for sensorless vector control of induction motor drives, Energy Conversion Congress and Exposition, pp. 2466-2473, 2009.

[11] G. Cimini, G. Ippoliti, G. Orlando and M. Pirro: Current sensorless solutions for PFC of boost converters with passivity-based and sliding mode control, Power Engineering, Energy and Electrical Drives, pp. 1175-1180, 2013.

[12] A. J. Koshkouei and A. S. I. Zinober: Sliding mode state observers for discrete-time linear systems, International Journal of Systems Science, Vol. 33, No. 9, pp. 751-758, 2002.

[13] S. K. Spurgeona: Sliding mode observers: A survey, International Journal of Systems Science, Vol. 39, No. 8, pp. 751-764, 2008.

[14] H. J. Yang, Y. Xia and P. Shi: Observer-based sliding mode control for a class of discrete systems via delta operator approach, Journal of the Franklin Institute, Vol. 347, No. 7, pp. 1199-1213, 2010.

[15] I. Haskara: On sliding mode observers via equivalent control approach, International Journal of Control, Vol. 71, No. 6, pp. 1051-1067, 2010.

[16] Y. Shtessel, C. Edwards and L. Fridman: Sliding Mode Control and Observation, Birkhauser, 2013.

[17] A. Sainana: A novel sliding mode observer applied to the three-phase voltage source inverter, European Conference on Power Electronics and Applications, pp. 1-12, 2005.

[18] F.M. Oettmeier, J. Neely, S. Pekarek, R. DeCarlo and K. Uthaichana: MPC of switching in a boost converter using a hybrid state model with a sliding mode observer, IEEE Trans. Indus. Elect., Vol. 56, No. 9, pp. 3453-3466, 2009.

[19] A. Jaafar, E. Godoy, P. Lefranc, X. Lin-Shi, A. Fayaz and N. Li: Nonlinear sliding mode observer and control of high order DC-DC converters, Annual Conference on IEEE Industrial Electronics Society, pp. 180-186, 2010.

[20] Z. Chen: Double loop control of buck-boost converters for wide range of load resistance and reference voltage, IET Control Theory and Applications, Vol. 6, No. 7, pp. 900910, 2012.

[21] S.-C. Tan, Y.M. Lai, C. K. Tse, L. Martinez-Salamero and 
C.-K. Wu: A fast-response sliding-mode controller for boost-type converters with a wide range of operating conditions, IEEE Trans. Indus. Elect., Vol. 54, No. 6, pp. 3276-3286, 2007.

[22] H. H. Choi and K.-S. Ro: LMI-based sliding-mode observer design method, Proc. Inst. Elect. Eng. Contr. Theory Appl., Vol. 152, pp. 113-115, 2005.

[23] A. J. Koshkouei and A. S. I. Zinober: Sliding mode control of discrete-time systems, Journal of Dynamic Systems, Measurement, and Control, Vol. 122, No. 4, pp. 793 - 802, 2000.

[24] V. Utkin and H. Lee: Chattering problem in sliding mode control systems, International Workshop on Variable Structure Systems, pp. 346-350, 2006.

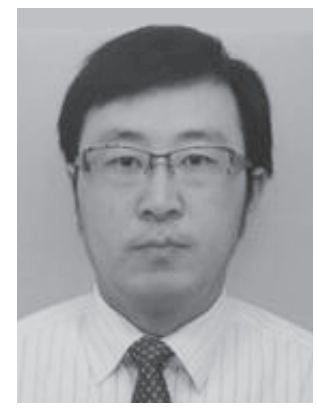

Xutao Li received his B.S. degree in automation from Wuhan Technology University, China, in 1999, and his M.S. degree in precise instruments and machinery from Shanghai Jiaotong University, China, in 2006. He is now a Ph.D. candidate in the Graduate School of Information, Production and Systems, Waseda University, Japan. His research interests include switching converters, robust $\mathrm{H} \infty$ control, and sliding mode control.

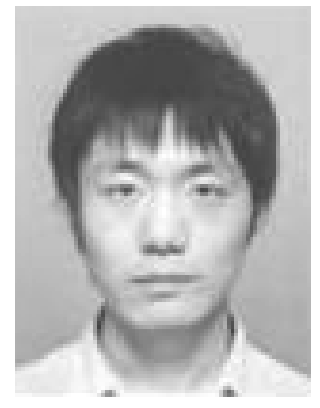

Minjie Chen received his B.S degree in electrical engineering and automation from Shanghai Jiao Tong University, China, in 2009. He received M.S. degree from the Graduate School of Information, Production and Systems, Waseda University, Japan. He is currently a Ph.D. candidate in the same school. His research interest is power converter technology.

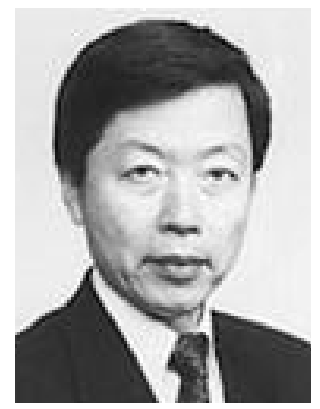

Tsutomu Yoshihara received his B.S. and M.S. degrees in physics and his Ph.D. degree in electronic engineering from Osaka University, Osaka, Japan, in 1969, 1971, and 1983, respectively. In 1971, he joined ULSI Laboratory of Mitsubishi Electric Corporation, Hyogo, Japan, where he was engaged in the research and development of MOS LSI memories. Since April 2003, he has been a Professor in the Graduate School of Information, Production and Systems, Waseda University, Fukuoka, Japan, and is currently involved in research on system LSI. Dr. Yoshihara is a member of IEEE Solid-State Circuits, the Institute of Electronics, Information and Communication Engineering (IEICE) of Japan, and the Institute of Electrical Engineers of Japan.

(Received January 19, 2015; revised Aprile 9, 2015) 